\section{LA DIGNIDAD DE LOS MÁRGENES. APROXIMACIONES AFECTIVAS A LA CIUDAD INFORMAL'}

Mónica Aubán Borrell²

\section{DIGNITY IN THE MARGINS. AFFECTIVE APPROACHES TO THE INFORMAL CITY'}

\author{
Mónica Aubán Borrell²
}

\section{Abstract}

La llamada teoría del afecto parece, hoy en día, muy alejada de la disciplina arquitectónica. Sin embargo, en los últimos años se están produciendo una serie de aproximaciones a las llamadas ciudades informales que nos permiten cuestionar este alejamiento. La incorporación de variables subjetivas como la experiencia de vida de los habitantes de las ciudades informales, consideradas elemento fundamental de proyecto urbano, nos permite situarnos muy cerca del afecto. En este sentido, el presente artículo sostiene que las propuestas enmarcadas dentro de la rearticulación socio-espacial que está planteándose en diversas ciudades
The so-called affect theory seems to be unrelated to architecture. However, the recent exploration of informal cities challenges this idea. The incorporation of subjective variables, such as the life experience of those living in informal cities -regarded as key elements for urban projects-, enable us to take a close look to the notion of affect. This paper argues that current proposals associated with socio-spatial reconfiguration in informal Latin American cities create a close relationship between architecture and the affect theory, more specifically with its French 
informales latinoamericanas permiten establecer un estrecho vínculo entre la práctica arquitectónica y la teoría afectiva; concretamente, con una rama de la teoría afectiva que viene del postestructuralismo francés, que otorga a las nociones de territorio y espacialidad un papel fundamental. Para ilustrar esta relación se presentan dos ejemplos de intervenciones concretas: la de Jorge Mario Jáuregui en Río de Janeiro y la de Urban-Think Tank en Caracas; dos ejemplos en los que la reflexión teórico-práctica y la preocupación por la implicación comunitaria sientan las bases para la inclusión de las variables afectivas en el ejercicio de comprensión y producción de territorios habitados.

PALABRAS CLAVE: CIUDAD INFORMAL, TERRITORIO, AFECTO, ACCIÓN COMUNITARIA, TEORÍA CRÍTICA.

\section{Recibido: 28-02-2017.}

Aceptado: 31-08-2017.

1 El presente trabajo es un resultado de la investigación doctoral en curso: El mito de la ciudad informal. Los afectos negativos en la producción y vivencia del espacio público. La tesis se está desarrollando en el marco de la ayuda predoctoral FI-DGR, que participa en programas del Fondo Social Europeo (FSE).

2 España. Doctoranda del Departamento de Teoría e Historia de la Arquitectura y Técnicas de la Comunicación de la Escuela Técnica Superior de Arquitectura de Barcelona (ETSAB), Universidad Politécnica de Cataluña. Correo electrónico: monica.auban@ upc.edu. post-structuralist branch, which acknowledges a key role played by the notions of territory and spatiality. This relationship is illustrated by two examples of concrete interventions: Jorge Mario Jáuregui, in Río de Janeiro and UrbanThink Tank, in Caracas. These cases show that theoretical-practical reflections and the concerns about community-based involvement lay the basis for the inclusion of affective variables into the understanding and production of inhabited territories.
KEYWORDS: INFORMAL CITY, TERRITORY, AFFECTION, COMMUNITY ACTION, CRITICAL THEORY.

Received: 28-02-2017.

Accepted: 31-08-2017.

1 This paper is part of an ongoing dissertation entitled "The Myth of the Informal City. Negative Emotions associated with the Production and Experiences in Public Spaces." This research is supported by the pre-doctoral FI-DGR fellowship, part of the European Social Fund (ESF.)

2 Doctoral student, Department of Theory and History of Architecture and Technical Communication at the Barcelona School of Architecture, Polytechnic University of Catalonia. Email: monica.auban@upc.edu. 


\section{FIGURA 1. VIVIENDAS EN VALPARAÍSO, JULIO DE 2016. FUENTE: AUTORA.}

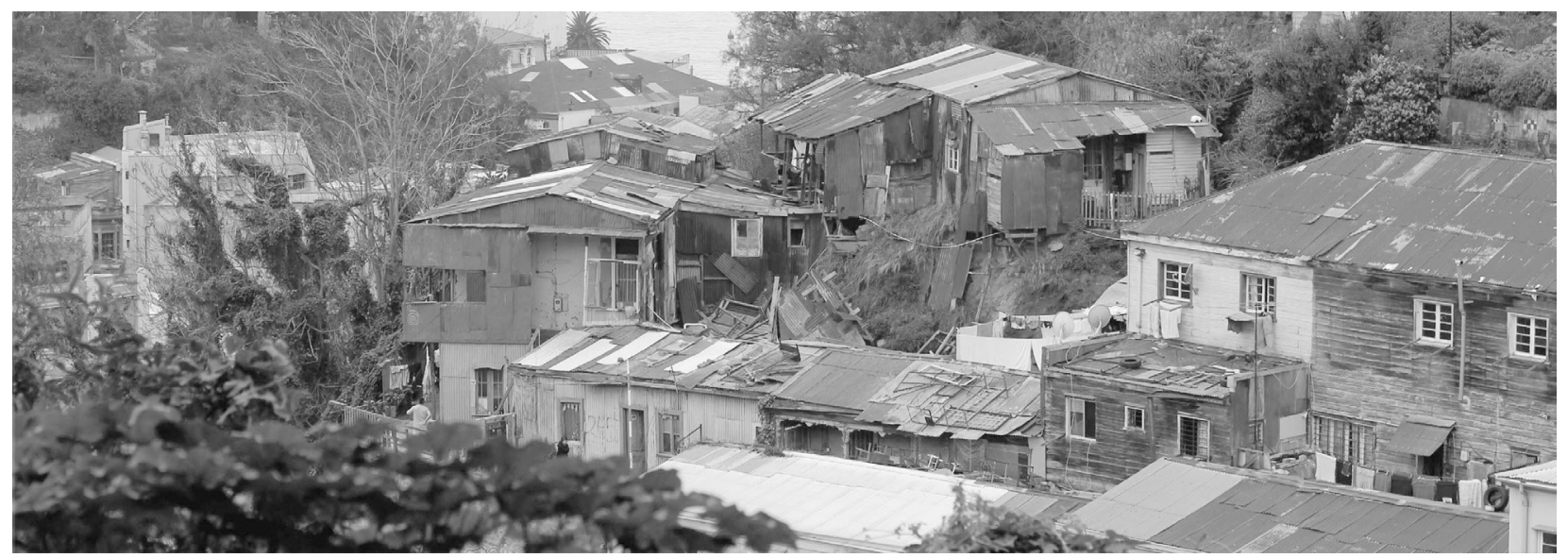

"Trepamos a pie por los cerros del puerto, llegando a la cúspide de la miseria desde donde se desciende a Valpore. Por un sendero en espiral que conduce a lo más bajo, pasamos frente a casas sin terminar en las que se acumulaban ladrillos, y niños que apenas sabian caminar jugaban a dispararnos. En un momento nos detuvimos, quisimos descansar, pero las piedras que nos lanzaban nos pusieron otra vez en camino hacia el plan de Valpore. La noche llegaba. La vista era la de un tornado descendente de luces; había que seguirlas para llegar al centro".

Valpore, Cristóbal Gaete. 


\section{Introducción}

Existe, en cada espacio habitado, una realidad subyacente que escapa a las características materiales y físicas que lo conforman. Una realidad que a veces es un paisaje y, otras veces, los recuerdos de una infancia. Una realidad que, en ocasiones, las palabras consiguen retener, al menos durante un breve instante. Así, entrevemos aquello que se oculta cuando pronunciamos la palabra casa, la palabra vivienda; aquello que se oculta al decir hogar, barrio, calle o periferia. $\mathrm{O}$, también, podríamos pensar en todo aquello que quisiéramos ocultar cuando decimos chabola; cuando decimos población, villa, pueblo joven o favela. Aquello mismo que la breve cita de Cristóbal Gaete insinúa al describirnos un cerro construido de miserias: la de los niños que aprenden a disparar antes de caminar, la de las casas inacabadas; esas miserias que quedan lejos del centro reconocido de las ciudades.

Si bien desde finales de los años noventa los llamados affect studies han fijado las directrices para incorporar a diferentes ramas de la investigación la experiencia derivada de la vivencia de las emociones o los afectos - una incorporación que, sin lugar a dudas, podría ayudar a desvelar esa realidad subyacente de la que hablamos-, la disciplina arquitectónica, en general, y los estudios urbanos, en particular, parecen haber desoído ampliamente las múltiples posibilidades que la incorporación del enfoque afectivo aportaría en los análisis de producción del territorio y del hábitat.

Pese a la situación descrita, el presente artículo sugiere que existen indicios de una cierta aproximación a cuestiones relativas al afecto en el seno de la disciplina arquitectónica. Estos indicios, como veremos, se observan en una dimensión arquitectónica muy específica: aquella que se refiere a las políticas de intervención sobre las llamadas ciudades informales. Desde la década de los noventa se han venido produciendo numerosos movimientos de reivindicación de las áreas informales como elementos integrantes, con toda legitimidad, de las ciudades de las que forman parte. Se inicia así un doble movimiento, de reconocimiento por un lado y de supresión del estigma por otro, que perfectamente puede ser explicado en términos afectivos.

Además, la situación de muchas de estas iniciativas en países como Brasil, Venezuela, Colombia o México nos permite centrar la discusión sobre la repercusión de las emociones y los afectos, precisamente, en el contexto latinoamericano.

Aunque, quizás, el enfoque desde el afecto que aquí estamos reclamando sea de carácter tangencial y en absoluto explícito, consideramos que las aproximaciones que en este sentido se están realizando desde la informalidad podrían llegar a suponer la apertura de una vía que permita la extensión de este enfoque afectivo a otras dimensiones 
de la arquitectura que no se limiten a la realidad fracturada de las periferias globales.

Por otra parte, la propia extensión de los estudios dedicados al afecto exige una aclaración en cuanto a los referentes a los que nos remitiremos para situar el marco teórico de la reflexión que vamos a presentar. Las líneas de investigación que emergen desde la teoría del afecto son muchas y muy variadas, teniendo cada una de ellas un foco de atención específico. La filosofía, la geografía, la política o las ciencias sociales han sabido encontrar en la incorporación del afecto a sus discursos un motor para la producción de teoría crítica.

Entre el amplio abanico de posibilidades, optaremos por situar nuestra reflexión en la línea de una corriente de pensamiento que parte de una facción del postestructuralismo francés; facción que, en los años ochenta del siglo pasado, decidió apostar por la recuperación del pensamiento del filósofo barroco Baruch Spinoza, uno de los primeros pensadores en dedicarse al estudio de los afectos. Concretamente, nuestro marco teórico va a estar sustentado sobre las figuras de Gilles Deleuze, componente de facto de esta corriente postestructuralista a la que hemos hecho referencia, y Rosi Braidotti, filósofa contemporánea de gran relevancia en materia de estudios de género. La justificación de esta elección se basa en que el establecimiento de relaciones entre afecto, territorio y contexto, por parte de estos autores, permite acercar el debate teórico de la afectividad a la práctica arquitectónica. Pero, antes de introducirnos en esta práctica arquitectónica, hemos de comprender cuál es la definición de afecto que estos dos pensadores proponen.

\section{Hacia una definición del afecto como corriente interpersonal}

El 25 de noviembre de 1980, el filósofo Gilles Deleuze iniciaba su seminario En medio de Spinoza, en la Universidad de Vincennes (Deleuze, 2015). Este seminario supuso el establecimiento de una línea de continuidad con el trabajo iniciado por Louis Althusser para devolver a su lugar en los planes de estudio de filosofía -si es que alguna vez lo tuvoa Baruch Spinoza. Esta recuperación de la figura del gran ateo del barroco marcó profundamente a toda una generación de influyentes intelectuales de la izquierda marxista, como Antonio Negri o Etienne Balibar.

En cualquier caso, el interés de Gilles Deleuze por la figura de Spinoza provenía de años anteriores. De hecho, su tesis doctoral (Spinoza o el problema de la expresión, publicada en el año 1967) estaba ya dedicada al estudio de este filósofo, y las referencias a su pensamiento son prácticamente una constante en toda su obra. Deleuze llegó a referirse 
a Spinoza como "el príncipe de los filósofos" y reconoció abiertamente que fue al pensador holandés a quien dedicó el mayor tiempo de estudio durante toda su carrera (Dosse, 2009, p. 187).

Como le ocurre con tantos otros autores, la lectura que Gilles Deleuze nos presenta de la obra de Spinoza deriva en una particular simbiosis en la que las ideas de ambos pensadores se entremezclan, dejando al lector confundido ante el tono deleuziano de Spinoza o viceversa. Y mejor viceversa pues al acudir a la fuente, el mismo lector se dará cuenta de que es Deleuze quien es radicalmente spinozista y que es también Deleuze quien hace hablar a Spinoza en su propia lengua. Es precisamente el uso de esta lengua lo que más nos interesa, ya que cuando Deleuze nos habla en ella, su mente parece estar siempre ocupada por geografías, espacios y territorios.

Entre los numerosos puntos de encuentro que pueden observarse entre estos autores, la atención prestada al estudio de los afectos, por parte de ambos, ocupa un papel fundamental. Así vemos cómo en la obra Ética, Spinoza dedica dos libros completos al análisis de los afectos, centrándose tanto en el origen y la naturaleza de éstos como en su influencia para el desarrollo del ser humano (Spinoza, 2015).

Debería sorprender, en este sentido, el papel protagonista concedido al afecto que nos es presentado como una pieza fundamental del andamiaje que sostiene el edificio racionalista que Spinoza erige con su Ética; una ética tan profundamente racional que ya desde su mismo título es introducida como "demostrada según el orden geométrico". No podemos olvidar que Baruch Spinoza desarrolla su pensamiento en un momento histórico en el que la filosofía continental estaba dominada por el espíritu cartesiano. Fue en el siglo XVII cuando la razón impuso su hegemonía como filtro absoluto del conocimiento y de la acción humana. Así, en un contexto dominado por el dualismo establecido entre los dos polos de una res cogitans (sustancia pensante) y una res extensa (sustancia extensa), Spinoza nos introduce en un mundo de inmanencia radical donde la emoción o los afectos son tan constitutivos del Ser como la capacidad de raciocinio que ya había sido encumbrada.

Para tratar de explicar el alcance de las ideas de Spinoza debemos, en primer lugar, preguntarnos a qué se refiere este autor cuando analiza los afectos. En el libro tercero de la Ética, en el apartado dedicado a las definiciones que Spinoza introduce al inicio de cada libro, leemos:

Por afecto entiendo las afecciones del cuerpo por las cuales aumenta o disminuye, es favorecida o perjudicada la potencia de obrar (Spinoza, 2015, p. 209-210).

$\mathrm{Y}$, aunque totalmente descontextualizada de la totalidad del libro, esta breve definición puede aportar ya muchas pistas sobre la noción de afecto 
que vamos a suscribir. En primer lugar, destaca la relación que Spinoza establece entre afecto y cuer$\mathrm{po}^{3}$-relación que tendrá profundas resonancias en las teorías del llamado giro afectivo-. En segundo lugar, la referencia directa en esta definición tanto a las afecciones como a la potencia anuncia ya un fuerte componente relacional del afecto.

Para comprender mejor esta componente relacional que caracteriza a los afectos, resulta necesario aclarar que bajo el término afecciones Spinoza hace referencia a las variaciones que modifican, de manera continuada, la conformación de nuestro cuerpo y de nuestra alma. Mientras que, por su parte, la potencia alude a la capacidad con la que contamos para pasar a la acción, es decir, se refiere a nuestra inclinación para actuar (Spinoza, 2015, p. 221).

Tanto las afecciones en su variación, como la potencia que, como vemos en la definición, aumenta o disminuye, es favorecida o perjudicada, nos remiten a una idea de gradiente. Spinoza define así una cosmología propia según la cual toda la realidad puede ser traducida en los términos de un sistema de fuerzas. Esta definición resulta

3 Debemos señalar que en Spinoza aparece una suerte de correspondencia entre el alma y el cuerpo, de manera que aquello que afecte al cuerpo afectará al alma y viceversa. Esta correspondencia, a la que autores posteriores llamarán "teoría del paralelismo", resulta fundamental para comprender la importancia que Spinoza otorga al cuerpo, al que sitúa al mismo nivel que el espíritu. fundamental, pues a partir de ella Spinoza nos introduce en un mundo en el que todas las cosas se encuentran interconectadas, relacionándose de manera permanente unas con otras y produciéndose de este modo cambios y transformaciones de manera continuada.

Es en este mundo de interconexiones donde la idea de afecto cobra fuerza, ya que los afectos son entendidos como el vehículo de estas transformaciones. Nos encontramos así frente a una definición del afecto por parte de Spinoza como capacidad para afectar y ser afectado. Una definición que, varios siglos después, tanto Gilles Deleuze como Rosi Braidotti harán suya plenamente para incorporarla en sus diferentes aportaciones a la teoría crítica.

Pero, antes de entrar en aproximaciones más contemporáneas, volvamos por un momento al siglo XVII para valorar la repercusión que tuvo la introducción del afecto por parte de Spinoza. Aunque a lo largo de todo el discurso de la ética Spinoza se mantiene firme en su defensa de la razón, su análisis de los afectos se refiere a cuestiones como el deseo, la alegría y la tristeza -considerada esta tríada como los tres afectos primarios de los que derivan 
todos los demás (Spinoza, 2015, p. 224-226)- o, también, el asombro, el desprecio, el amor, el odio, la repulsión, el miedo, la indignación... Nos encontramos así con una larga lista de sensaciones que apelan directamente a la subjetividad.

Esta referencia a la dimensión subjetiva dentro de un sistema marcadamente racionalista supone un gesto intelectual de enorme transcendencia. El foco de interés que Spinoza sitúa sobre los afectos implica la apertura de una brecha en un contexto dominado por la pretensión de una objetividad pura. El imperio, casi tiránico, de la razón como verdad única supone relegar a un segundo, o a un tercer plano, todos aquellos impulsos relacionados con la percepción, la sensación, la imaginación o el deseo. De hecho, esta situación en planos inferiores de interés sigue muy vigente hoy en día, por ejemplo en la obsesión por encontrar explicaciones científicas, desde la biología, la neurología o incluso la psicología, que den cuenta del origen de las emociones. Así como Spinoza elevó el cuerpo para situarlo al mismo nivel que el alma o, si prefiere, que el espíritu, la emoción y el afecto son devueltos al mismo plano de la razón y el pensamiento.

Aunque tendrán que pasar muchos años para que estas ideas sean retomadas, es precisamente en esta brecha abierta por Spinoza donde debemos situar el interés de Gilles Deleuze por este autor. Y es también en esta fractura abierta donde podemos empezar a instalar el interés de los afectos para la disciplina arquitectónica.

Resulta del todo evidente que la práctica arquitectónica ha privilegiado, tradicionalmente, los enfoques de objetivismo, o pretendido cientifismo, por encima de posibles aproximaciones que se ocuparan de la vivencia subjetiva. En este sentido observamos cómo el proyecto, ya sea edilicio o urbano, está comúnmente regido por razones formales, geométricas, materiales o económicas que desatienden la importancia de la experiencia asociada a la acción de recorrer, habitar u ocupar el espacio construido.

La puesta en valor de los afectos por parte de Spinoza y su consideración como componentes fundamentales del Ser, pueden ser conceptos adoptados como un buen punto de partida para empezar a acortar las diferencias entre el ejercicio de proyección apriorístico de la arquitectura y la posterior experiencia del espacio habitado.

Pero volvamos a situarnos en la línea de pensamiento de Gilles Deleuze. Con independencia del diálogo establecido con la obra de Baruch Spinoza, la noción de afecto cuenta con una presencia propia en la trayectoria de este filósofo. Aparecen, en este sentido, diferentes aproximaciones a la noción de afecto que van matizándose y reformulándose según la evolución de sus escritos. Pese a esto, es 
posible encontrar un nexo común que aúna todas estas aproximaciones bajo una definición de afecto que va de la mano de los devenires deleuzianos y que conecta el afecto con las modificaciones, transformaciones y cambios a los que la realidad, en sus diferentes dimensiones, tangible e intangible, se ve sometida de manera permanente (Parr, 2010, p. 11-14).

Por otra parte, la adscripción a la conceptualización spinozista del afecto como capacidad para afectar y ser afectado deriva en Deleuze en la comprensión del afecto como una corriente interpersonal que atraviesa y recorre los cuerpos, provocando la emergencia de diferentes sistemas de fuerzas y relaciones. Esta comprensión del afecto implica, necesariamente, la participación de al menos dos cuerpos o entidades en la relación que va a provocar el cambio o la transformación. Subyace, en este sentido, una componente colectiva en esta aproximación al afecto, que pensadores como Deleuze, Rosi Braidotti o Brian Massumi van a reivindicar con fuerza.

De hecho, la idea de colectividad es fundamental en la relectura del concepto de afecto de Spinoza por parte de Rosi Braidotti. Su situación teórica en la línea de pensamiento de la diferencia sexual inicia un cuestionamiento acerca de la conformación de la subjetividad en el contexto contemporáneo; un cuestionamiento en el que las minorías -con un papel especial para las mujeres- juegan un papel fundamental. Existe en Braidotti una preocupación recurrente por el efecto de las interconexiones mediadas por la tecnología, a las que la materia subjetiva se ve expuesta recurrentemente. El afecto, que alcanza aquí un fuerte contenido socio-político, es entendido como el impulso que articula estas relaciones entre diferentes agentes (humano, animal, insecto, cyborg...) y su medio (Braidotti, 2009, p. 22-25). El enfoque situado que defiende Braidotti, es decir esta toma de conciencia en cuanto a las coordenadas espacio-temporales concretas que conforman nuestra subjetividad en su movimiento de transformación constante, posee también un gran potencial para su aplicación en la práctica arquitectónica.

A lo largo del presente artículo se seguirá esta línea de pensamiento que entiende el afecto como una corriente interpersonal que sacude a la subjetividad, moviéndola a la acción. Para su extrapolación al campo arquitectónico, la componente de colectividad que ha sido explicada se convierte en un valor fundamental, ya que resulta imposible imaginar una vivencia de un territorio que no sea colectiva. La realidad urbana que los territorios habitados conforman es inseparable del complejo conjunto de relaciones e intercambios que en ella tienen lugar (Lefebvre, 1967, p. 59). La aproximación desde el afecto a esta suerte de territorialidad vivida puede ser entendida como un buen mecanismo para empezar a desvelar e incorporar estos 
juegos de relaciones constitutivas en los propios procesos de la práctica arquitectónica.

\section{Cualidades intensivas del espacio: el territorio apropiado}

Esta referencia al territorio nos sirve ya para anunciar la conexión entre arquitectura y afecto que estamos buscando, una conexión que es inseparable de la componente territorial común a ambas entidades. Para explorar esta conexión debemos volver a recuperar el discurso de Gilles Deleuze; concretamente, debemos recuperar su interés por la geografía y el territorio que aparece de manera constante a lo largo de toda su obra, hasta el punto de que Deleuze llega a ocuparse de una conceptualización espacial propia, inseparable de la noción de afecto.

Sin duda, una de las expresiones más relevantes de este interés por la geografía y el territorio lo encontramos en la obra conjunta de Gilles Deleuze y Félix Guattari. De hecho, el título Mil mesetas, elegido para el segundo volumen de la serie Capitalismo y esquizofrenia, da, por sí solo, buena cuenta de ello. Es precisamente en este libro donde Deleuze y Guattari introducen esa conceptualización del espacio que es profundamente afectiva.
Así, en la Meseta -o capítulo 14-, del libro: 1440 Lo liso y lo estriado (Deleuze y Guattari, 1980, p. 483-509), nos topamos con dos formas de espacialidad claramente diferenciadas: el espacio liso y el espacio estriado; dos formas que se corresponden, a su vez, con diferentes mecanismos para la apropiación del territorio.

En cuanto a una primera definición de estas dos espacialidades, Deleuze y Guattari expresan:

El espacio liso está ocupado por acontecimientos o haecceides, mucho más que por cosas formadas o percibidas. Es un espacio de afectos más que de propiedades. Es una percepción háptica más bien que óptica. Mientras que en el estriado las formas organizan una materia, en el liso los materiales señalan fuerzas o le sirven de síntomas. Es un espacio intensivo más bien que extensivo, de distancias y no de medida. Spatium intenso en lugar de Extensio. (Deleuze y Guattari, 1980, p. 487).

Resuena todavía en esta definición un cierto malestar por la imposición del espacio único cartesiano, que intenta ser superada. Para ello, las propiedades cuantificables y medibles del espacio extenso -espacio estriado, en términos de los propios autoresson completadas con una serie de cualidades intensas y difícilmente mesurables que componen el llamado espacio liso. En varias ocasiones a lo largo del capítulo, esta forma de espacialidad lisa viene ilustrada como un territorio ocupado por vientos, 
ruidos, fuerzas, cualidades táctiles y sonoras (Deleuze y Guattari, 1980, p. 487). El espacio liso es el del desierto y de la estepa, pero también -y esto resulta fundamental para nuestra reflexión- es el espacio de las ciudades.

Los ejemplos que ilustran la forma de espacialidad lisa apuntan, de manera directa, a la definición ampliada que Deleuze y Guattari tienen de la idea de territorio. Para estos pensadores, un territorio nunca podrá ser limitado a sus características meramente físicas o geográficas. En ¿Qué es la filosofía?, otro de sus trabajos conjuntos, exponen:

Hay que ver cómo cada cual, en todas las épocas de su vida, tanto en las cosas más nimias como en las más importantes pruebas, busca un territorio, soporta o emprende desterritorializaciones y se reterritorializa casi sobre cualquier cosa, recuerdo, fetiche o sueño. Los estribillos de las canciones expresan estos poderosos dinamismos: mi casita en Canadá... adiós me voy... sí, soy yo, tenía que volver (Deleuze \& Guattari, 2013, p. 69).

Aunque la terminología de estos pensadores puede resultar, en ocasiones, confusa, en una primera aproximación podemos entender las acciones de desterritorializar y reterritorializar como diferentes ejercicios de apropiación de territorios; unos ejercicios que pueden ser tanto personales como colectivos. En cualquier caso, lo que nos interesa señalar es cómo la noción de territorio, en las tesis de Deleuze y Guattari, se expande abarcando dimensiones que se sitúan mucho más allá de lo material y de lo tangible. Es a partir de esta noción ampliada cuando resulta posible establecer conexiones entre territorio y afecto.

Para Deleuze y Guattari, un territorio determina siempre la aparición de cualidades sensibles (2013, p. 186), que, como veíamos, dependen del viento, del ruido o del tacto, y que, sobre todo, tienen la capacidad de afectar al medio habitado de manera integral -incluyendo aquí también a los ocupantes de ese medio-, provocando su alteración o transformación. Lejos de configurarse como una representación estática del territorio, la lectura desde el afecto nos permite su reconocimiento como una entidad dinámica y compleja, cuya realidad está sometida al impacto de miles y miles de sistemas de relaciones que van tejiendo una red de frágiles equilibrios susceptibles de ser disueltos en cualquier momento.

Esta identificación del territorio como entidad dinámica e inestable es fundamental para la práctica arquitectónica. La producción de hábitat y de territorios, de los que tanto la arquitectura como el urbanismo se encargan, participa profundamente de este sistema de relaciones que caracteriza a cada entorno concreto. Pese a esto nos topamos frecuentemente con la pretensión, por parte de la disciplina, de que la acción arquitectónica puede llegar a "ordenar" o "regular" estos sistemas de relación 
inestable. Evidentemente, la influencia afectiva no puede nunca funcionar unidireccionalmente, y el proyecto, una vez construido, queda inmerso en este mismo sistema de relaciones y está sometido a unas variaciones difícilmente predecibles. Sin embargo, esta situación no debería suponer un freno al ejercicio proyectual. Al contrario, la consideración de estas variables afectivas desde las propias fases de análisis de los proyectos podría ayudar a proponer soluciones mejor adaptadas a las demandas de cada territorio concreto.

Retomando el discurso de lo liso y lo estriado, según un movimiento que es típico en Deleuze y Guattari, las relaciones que estos autores observan entre el espacio liso y el espacio estriado no se limitan a la mera confrontación. Lo liso y lo estriado cohabitan y conviven en los mismos lugares, desatándose entre ellos un flujo continuo de tensión que pugna por estriar u ordenar lo liso y que deshace y diluye lo estriado (Deleuze \& Guattari, 1980 , p. 483). En este sentido, resulta muy interesante profundizar en la identificación, por parte de estos dos autores, de la ciudad como un espacio paradigmático de la conflictiva convivencia de lo liso y lo estriado.

Contrariamente al mar, la urbe es el espacio estriado por excelencia; pero así como el mar es el espacio liso que se deja fundamentalmente estriar, la urbe será la fuerza de estriaje que volvería a producir, a abrir por todas partes espacio liso, en la tierra y en los demás elementos -fuera de ella, pero también en ella- (Deleuze \& Guattari, 2012, p. 489-490).

La ciudad, la urbe, es reconocida como el sitio privilegiado del estriaje. Indudablemente, la ciudad es el escenario sobre el que se inscriben las políticas de planificación. Es el espacio de la reglamentación y las normativas para la calificación de los usos del suelo, la definición de las alturas de la edificación, el diseño de la zonificación, las regulaciones ambientales y un largo etcétera que conforma el campo legislativo de la disciplina del urbanismo. Pero, a su vez, la ciudad es también el espacio de lo imprevisto, de la sorpresa, de la fiesta y de la revuelta urbana; la ciudad es además el espacio de lo posible. Es en este doble sentido en el que la ciudad se convierte en una constatación de la disputa entre las fuerzas de lo liso y de lo estriado. Pese a todas las normas que los técnicos y los planificadores puedan pensar para la ciudad, siempre habrá en lo urbano algo que se escape, algo capaz de resistir a la normalización impuesta.

La situación del análisis de la realidad urbana desde la perspectiva del afecto implica una puesta en valor del conjunto de variables intensivas -o variables imprevisibles- que también recorren el espacio de la ciudad. Las acciones de reterritorialización y desterritorialización a las que Deleuze y Guattari hacen referencia, representan muy bien esta doble caracterización cualitativa-cuantitativa 
que persiste en las ciudades y que permite a sus habitantes la apropiación selectiva de determinados lugares o espacios para una nueva re-significación colectiva.

Esta identificación de la ciudad como un territorio de ambivalencia entre cualidades sensibles y objetivas adquiere un valor fundamental si nos trasladamos al contexto de lo informal. En Mil Mesetas, los propios Deleuze y Guattari señalan a los suburbios como un vector de fuerza del espacio liso capaz de irrumpir en medio del entramado estriado de las ciudades (Deleuze \& Guattari, 2012, p. 489-490).

Desde una perspectiva arquitectónica, resulta muy fácil reconocer ese punto de ambivalencia que existe en la informalidad. Las llamadas ciudades informales se constituyen hoy en día como uno de los fenómenos más potentes de los procesos de urbanización global y, sin embargo, isería posible encontrar una tipología urbana más alejada de los sistemas de planificación vigentes?

La realidad de las ciudades informales aparece hoy en día surcada por una serie de dinámicas propias que hacen difícil su adscripción a los valores de la tradición urbana canónica. Su situación tanto física como sociológica en el borde de las ciudades consolidadas, su característico estar al margen, las convierte en un elemento urbano de radical alteridad, cuya correcta lectura requiere nuevas maneras de análisis. Como hemos venido anunciando, el presente artículo defiende la idea de cómo una lectura afectiva del territorio puede ayudar a encontrar estos nuevos focos desde los que analizar y profundizar en esa realidad estigmatizada que las ciudades informales componen.

\section{La rearticulación socio- espacial. Nuevas dinámicas de intervención sobre lo informal}

La aproximación a la informalidad que vamos a iniciar debe situarse en un punto de partida que nos permita reconocer las características específicas de este fenómeno urbano. En este sentido debemos señalar que, aunque han sido ampliamente ignoradas por la tradición disciplinar de la arquitectura, las ciudades informales cuentan ya con una historia propia de evolución, que reconstruiremos de una manera breve.

Antes de comenzar, procede destacar que el desarrollo histórico de este fenómeno arquitectónico se encuentra fuertemente vinculado a la relación que las áreas informales han podido ir estableciendo con sus núcleos urbanos consolidados más próximos, generalmente por vía de la mediación de 
distintos organismos institucionales. En este sentido resulta posible identificar distintas fases en la relación formal-informal, en las que las políticas públicas de intervención desarrolladas sobre las áreas informales han ido modelando la realidad de estos asentamientos hasta alcanzar su situación actual. Desde las primeras muestras de hacinamiento en las periferias de las ciudades por el reclamo de la industrialización hasta las propuestas actuales de pacificación en varias favelas de Rio de Janeiro, es posible observar diversos movimientos en los términos de esta relación, en los que, poco a poco, la posibilidad de una lectura afectiva asociada a la informalidad ha ido abriéndose paso.

Tal y como señala Davis en su libro Planeta de ciudades miseria, la primera aparición del término slum tuvo lugar en 1812 refiriéndose en exclusiva a un tipo de comercio que crecía fuera de los sistemas regulados de mercado. En la década de 1830 a 1840, la palabra slum será ya ampliamente utilizada para designar a las áreas hacinadas que empezaban a consolidarse como núcleos de pobreza urbana (Davis, 2014, p. 34-35).

El posterior desarrollo de las áreas informales estuvo fuertemente determinado por el abandono absoluto por parte de las autoridades, que tuvo lugar durante las primeras décadas de existencia de este fenómeno urbano. El abandono institucional derivó en un proceso de consolidación de las áreas informales, según el cual la urbanización masiva e irregular que iba extendiéndose al amparo de los núcleos industriales creció sin encontrar un límite, más allá de las propias condiciones de vida hiperdegradadas que iban extendiéndose por estos asentamientos.

Hacia la década de los cincuenta del siglo XX, la situación de las áreas informales se vuelve insostenible. El problema de la vivienda empieza a imponerse, en estos años, como una necesidad urgente en muchas agendas internacionales, y comienzan a producirse, de manera sistemática y al amparo de los discursos sobre seguridad e higiene, movimientos de desalojo y realojos masivos de los habitantes informales. Las operaciones de urbanización y erradicación serán las estrategias dominantes durante varias décadas.

Pese a los intentos de erradicación de los asentamientos hiperdegradados, la urbanización informal seguirá creciendo de manera imparable. La crisis mundial de la deuda y los programas de desregulación agraria que tuvieron lugar en la década de los setenta supusieron un aumento del número de habitantes informales en las periferias globales (Davis, 2014, p. 25-32). En general, cualquier solución propuesta resultaba insuficiente, de modo que la brecha entre lo formal y lo informal iba haciéndose cada vez más grande. No solo es que los habitantes informales no tuvieran acceso a los mismos servicios con los que contaban los núcleos urbanos consolidados, sino que también una marca 
de estigma y exclusión social se iba apoderando de los asentamientos hiperdegradados.

El éxodo masivo de población rural hacia los núcleos urbanos produjo un desbordamiento de la acción política de los poderes y gobiernos de los países más pobres, quienes relegaron el problema de la informalidad a organismos internacionales (Davis, 2014, p. 97). En este contexto, la ONU (Organización de las Naciones Unidas) funda en 1972 UN-Habitat, el organismo que tiene por objeto la evaluación, estudio y análisis de los asentamientos humanos. Junto a UN-Habitat, el FMI (Fondo Monetario Internacional) y el BM (Banco Mundial) han ido delineando, institucionalmente, el pulso de la relación entre gobiernos locales y asentamientos informales.

Paralelamente, y desde el campo teórico, en los años setenta apareció también un interés hacia la realidad de las ciudades informales; un interés que estuvo principalmente motivado por el reconocimiento y la puesta en valor de las posibilidades de autogestión y autoconstrucción. Destacan, en esta línea, las aportaciones del arquitecto británico John F.C. Turner, quien, tras su experiencia en Perú y desde su ideología anarquista, va a convertirse en un defensor a ultranza de los valores de la informalidad.

Tanto las estrategias de urbanización y erradicación, como la toma de control por parte de organismos internacionales, supusieron una reducción progresiva de la influencia de los habitantes de las áreas informales en la toma de decisiones sobre su propio entorno. La nueva puesta en valor de la autogestión como una cualidad propia de lo informal -a la que se le llegó a suponer incluso la posibilidad de erradicar el problema de la vivienda (Turner, 1977) - trajo consigo, en cambio, una revalorización del papel de los habitantes, que eran reconocidos de pronto como agentes legítimos de su ciudad. Como veremos más adelante, esta consideración de los habitantes de lo informal como pieza fundamental del territorio va a ser vital para la incorporación del afecto en la práctica arquitectónica. La introducción de la dimensión subjetiva como un elemento relevante del proyecto urbano llegará a convertirse en un factor decisivo del éxito o del fracaso de las políticas públicas de intervención urbana.

Sin embargo, la consideración positiva de la experiencia de habitar lo informal no iba a durar mucho. Vemos así cómo, desde los años ochenta, la puesta en valor de los mecanismos de autoconstrucción quedó atrapada en las redes del sistema neoliberal, que desplazó el interés de la autogestión hacia la posibilidad de impulsar un supuesto capital informal que sería activado mediante la creación de títulos de propiedad (Davis, 2014, p. 98-100). La entrada del sector privado en el campo de la informalidad tuvo como consecuencia un notable aumento de la desigualdad, que sigue todavía muy vigente. 
Para acabar de completar el cuadro que nos permita situar mejor las intervenciones más recientes sobre lo informal, debemos atender al llamado giro participativo. En el año 2003, UN-Habitat publicó su informe The Challenge of Slums, donde las iniciativas de conservación con mejoras aparecen recogidas como la manera más adecuada de intervención sobre lo informal (UN-Habitat, 2003). El cambio operado en esta dirección venía anunciándose aproximadamente desde la década anterior. Los noventa fueron años en los que diferentes ONGs consolidaron una intensa actividad en las áreas informales que, aunque con muchos matices conflictivos como el propio Mike Davis señala (2014, p. 104-109), posibilitó un trabajo bastante directo con las comunidades afectadas por los problemas derivados de la informalidad.

El trabajo realizado in situ con estas comunidades abrió una vía para un tipo de intervención mucho más comprometida con las demandas específicas de los territorios informales, que es la que aquí vamos a explorar.

La mera denominación de las nuevas directrices de intervención con la expresión "conservación con mejoras" sugiere, por sí sola, un replanteamiento en cuanto a la consideración de los núcleos informales. Concretamente, la voluntad de conservación pone de manifiesto el reconocimiento de una serie de cualidades en la realidad informal que merecen ser mantenidas.
Este gesto de reconocimiento adquiere una enorme transcendencia. Desde sus inicios, los asentamientos informales han sido considerados como un fallo o anomalía de los sistemas de planificación dominantes, que debían ser eliminados. De hecho, las operaciones de erradicación, desalojos forzosos o, incluso, urbanización apuntan, todas ellas, en esta dirección. Empezar a identificar en las áreas informales unas características propias que deben ser conservadas implica empezar a deshacer esa relación formal-informal, según la cual lo formal se impondría como solución hegemónica dominante. Evidentemente, la identificación de ciertas cualidades en los territorios informales no debe entenderse, en ningún caso, como una negación de los muchos problemas y deficiencias estructurales que persisten en estos lugares. Se trata, más bien, de empezar a pensar en la informalidad como una realidad única que necesita de unos procedimientos propios para ser explorada y comprendida adecuadamente.

El intento de reformular esta relación, que desde varios sectores ya se está llevando a cabo, persigue un ambicioso ejercicio de integración entre las áreas informales y los núcleos urbanos consolidados, de manera que los primeros puedan librarse de la marca de exclusión que les ha sido impuesta.

Este ejercicio de integración se revela aún más complejo al considerar la negatividad discursiva que acompaña la emergencia de la informalidad. 
Nos encontramos, en este sentido, con una potente narrativa peyorativa que sobrevuela los territorios informales. De hecho, los relatos sobre delincuencia, peligrosidad, violencia, falta de salubridad y tráfico de drogas, que se extienden como el modelo de vida en estos territorios, conforman la imagen de lo informal que es comúnmente percibida.

Ante las dificultades de la situación descrita, la incorporación de los habitantes en los ejercicios de integración vuelve a despuntar como factor esencial para la reformulación de la informalidad. Por un lado, las cualidades que deben ser mantenidas se refieren, en muchas ocasiones, al estrecho vínculo que llega a producirse entre los habitantes de las propias áreas informales y su entorno. En este sentido podemos pensar, por ejemplo, en la común reticencia por abandonar áreas informales habitadas en condiciones de degradación o peligro. Volvemos a encontrarnos aquí con unas nociones de casa, barrio o territorio que sobrepasan con mucho la mera definición material o física, y que abren una vía hacia una posible lectura afectiva. Por otro lado, son los propios habitantes los únicos que, mediante el relato de su propia experiencia, pueden empezar a transformar la imagen negativa que sobre sus territorios se ha consolidado, reduciendo así la distancia física y psicológica que los separa de los centros de las ciudades.

De esta manera, la integración que se busca entre las áreas informales y los núcleos urbanos consolidados es inseparable de un tipo de intervención doble, sustentado en la rearticulación socio-espacial de los territorios fracturados. Emerge entonces un tipo de práctica arquitectónica que, acompañada por una definición ampliada de la noción de territorialidad, intenta incorporar el mayor número de agentes y factores en la acción urbana. La adscripción a este tipo de proyectos de cuestiones como el sentimiento de pertenencia, la identificación con el lugar o el reclamo por la dignidad apelan directamente a esa dimensión afectiva que subyace, también, en la realidad arquitectónica.

\section{La casa y el barrio: dignidad reclamada}

Para ilustrar, un tanto mejor, las aproximaciones a la realidad informal que desde el enfoque de las tácticas para la rearticulación socio-espacial se están llevando a cabo, introduciremos a continuación dos casos representativos de esta línea de acción, ambos ubicados en el contexto latinoamericano: las intervenciones del arquitecto Jorge Mario Jáuregui en las favelas de Rio de Janeiro y el trabajo del equipo venezolano Urban-Think Tank en la ciudad de Caracas.

Resulta necesario señalar que los dos casos seleccionados comparten un ejercicio de reflexión 
teórica que acompaña a las intervenciones. Y es precisamente esta reflexión teórica la que nos permite poner en comunicación las acciones desarrolladas en el plano arquitectónico con la teoría del afecto que hemos venido anunciando.

Analicemos, en primer lugar, el caso de Rio de Janeiro. Las intervenciones de Jorge Mario Jáuregui sobre el territorio de las favelas aparecen enmarcadas en el contexto de tres programas institucionales que la ciudad carioca ha acogido: Favela-Bairro, que se inició en el año 1994, PAC (Programa de Aceleraçao do Crescimento), iniciado en 2007, y, por último, el programa Minha Casa, Minha Vida, que tuvo su origen en el año $2009^{4}$.

De una manera general, podemos afirmar que el hilo conductor de las intervenciones desarrolladas por el equipo de Jorge Mario Jáuregui se sustentan sobre esa voluntad de integración de las favelas como agente activo de la ciudad que hemos mencionado anteriormente. Si bien las favelas son sin duda una figura icónica de la geografía de Rio de Janeiro, su exclusión de las infraestructuras, servicios y vida de la ciudad es clamorosa. La particular apuesta de Jáuregui no se basa solo en defender el derecho a la participación ciudadana de los habitantes de la favela, sino que además sus propuestas parten de un fuerte posicionamiento previo que entiende que, se quiera o no,

4 Para una aproximación gráfica a la obra de Jorge Mario Jáuregui puede consultarse: http://www.jauregui.arq.br/. las áreas informales son una parte constituyente de la ciudad de Rio de Janeiro (Jáuregui, 2012, p. 54) y que, como tal, deben ser consideradas según sus propios términos de evolución, desarrollo y demandas.

Este posicionamiento previo de Jorge Mario Jáuregui deriva en un reconocimiento de la favela como una tipología urbana propia que cuenta con sus propios códigos y su propia lógica. Develar estas lógicas internas será uno de los principales objetivos del equipo de Jáuregui.

En cuanto a la manera de proceder, el foco de las diversas intervenciones se centra sobre la producción de espacio público. En este sentido, en la reflexión de este arquitecto aparece una definición ampliada del espacio público que no se limita a la dotación de infraestructuras y servicios (Jáuregui, 2012, p. 126). La noción de espacio público es presentada, en cambio, como el sustrato que sostiene y posibilita la interacción social.

La preeminencia de la autoconstrucción en el contexto informal dibuja un paisaje en el que la vivienda se impone como tipología. La imposibilidad de acometer las tareas para la producción de este espacio público individualmente aleja la posibilidad 
de crear esos lugares de encuentro sobre los que se sustenta la vida social.

Esta importancia concedida al espacio común adquiere, en términos afectivos, una relevancia capital. En ella descansa la posibilidad de iniciar el reconocimiento del territorio de la favela como un lugar propio. El asfaltado de las calles, la creación de equipamientos y sitios de reunión, el poner nombre a las calles o número a las viviendas va, poco a poco, iniciando un proceso de transformación en la percepción de la favela, que empieza a ser vista como un barrio.

Para iniciar el cambio en la percepción es fundamental la implicación de los habitantes en el proceso de transformación y por ello la participación comunitaria es una táctica fundamental del proyecto:

A través de todo el proceso (desde el diseño hasta la ejecución de la obra), la interacción con la comunidad se va transformando.

Primero, la participación se caracteriza como la interlocución que permite definir el programa. En segundo lugar, con el desarrollo del proyecto, la participación de los residentes llega a ser más específica, al punto de tener la comunidad como consultora para la elaboración de los trabajos. Durante la construcción de la obra, mínimamente el $40 \%$ de la mano de obra contratada es parte de la comunidad (la empresa constructora tiene la obligación de que así sea). Finalmente, los miembros de la comunidad integran el equipo de POUSO (Puesto de Orientación Urbanístico y Social), que es una estructura pequeña que el municipio incluye en la comunidad, con la intención de supervisar los resultados de la intervención y como soporte para las adaptaciones futuras (Jáuregui, 2012, p. 97).

Se observa una preocupación por la integración de los habitantes en el diseño y construcción de su propia ciudad, que abarca desde las etapas previas a la ejecución hasta el seguimiento del proyecto cuando está ya en uso. Desde el campo de los afectos, la posibilidad de participar en la construcción y en la mejora del propio entorno desata, en los habitantes, un fuerte sentimiento de identificación con el lugar, que empieza a ser vivido como algo propio. Conseguir la activación de los habitantes, que se sientan parte fundamental del territorio que les envuelve, es una de las piezas claves sobre las que descansa el éxito o el fracaso de las intervenciones propuestas.

Otra característica fundamental de la metodología de Jorge Mario Jáuregui reside en el enfoque interdisciplinar que orienta los proyectos. El equipo de trabajo cuenta con la colaboración de arquitectos, ingenieros, trabajadores y educadores sociales. En el equipo hay diferentes sensibilidades acerca de la ciudad, pero todos sus integrantes entienden que cualquier acción urbana que no vaya acompañada 
de una voluntad de integración de la dimensión social quedará incompleta.

La referencia a la participación comunitaria nos permite establecer el nexo con las acciones promovidas por el estudio Urban-Think Tank (U-TT). Situado en Venezuela y dirigido por los arquitectos Alfredo Brillembourg y Hubert Klumpner, este estudio ha encontrado en la investigación teóricopráctica en torno a la informalidad el motor de su acción arquitectónica ${ }^{5}$. El escritor Justin McGuirk, en su recorrido radical por distintas ciudades, comenta a este respecto:

En 2003, U-TT lanž́ su primer proyecto de investigación ya bien financiado en los barrios de Caracas, invitando a artistas internacionales como Marjetica Potr a colaborar en proyectos, entre ellos el del retrete seco. Los resultados se convirtieron en Informal City, que sigue siendo uno de los mejores y más accesibles libros sobre la informalidad en Latinoamérica. En él se plantea claramente que los barrios no eran solo un problema a la espera de una solución, sino sistemas extraordinariamente autorregulados, lugares con virtudes. Sobre todo, eran realidades que existían sobre el terreno, una condición urbana demasiado generalizada como para que se pudiera revertir (McGuirk, 2015, p. 151).

5 Un panorama más completo de las actuaciones de estos arquitectos puede encontrarse en: http://u-tt.com/.
Efectivamente, uno de los grandes aciertos de UTT ha sido el de reconocer a los barrios como una pieza clave para la definición del desarrollo del urbanismo global en los próximos años (Brillembourg, Feireiss y Klumpner, 2005, p. 12). Así, la informalidad se presenta para este equipo como un enorme laboratorio de acción urbana que, en un contexto como el latinoamericano, requiere de nuevas lecturas capaces de develar las complejidades y particularidades propias.

Actuaciones como la de la Torre de David (UrbanThink Tank, 2013) ponen de manifiesto una cierta independencia de las instituciones, que suelen aparecer cuando la acción comunitaria ya está perfectamente articulada. La aproximación de U-TT a la práctica arquitectónica se caracteriza, en este sentido, por un respetuoso acercamiento a estas comunidades implicadas con cada entorno concreto, para entender la experiencia de habitar lo informal de la mano de sus propios habitantes.

La investigación que U-TT pone en marcha se inicia desde el propio cuestionamiento del término informal, que apela, directamente, a un cuestionamiento sobre la naturaleza del fenómeno. Se desprende de esta investigación un reclamo por encontrar herramientas de planificación alternativas, 
definidas explícitamente como no cartesianas (Brillembourg, Feireiss y Klumpner, 2005, p. 19). Recordemos aquí las aportaciones de los autores que hemos situado como marco teórico.

La búsqueda de estas herramientas alternativas de planificación entronca directamente con el reconocimiento de unas cualidades características de lo informal, que también encontrábamos en Jorge Mario Jáuregui. Y, nuevamente, la relación con los habitantes es la que permite sostener esta búsqueda.

Podemos destacar, en este sentido, la afirmación del líder comunitario del barrio de la Vega, Francisco Pérez: "Lo que ustedes llaman barrio es mi casa" (Brillembourg, Feireiss y Klumpner, 2005, p. 38). Detrás de esta simple frase podemos percibir la brecha ideológica que separa las áreas consolidadas de los núcleos informales.

Tratando ahora de hallar los nexos entre las actuaciones de los dos equipos mencionados, debemos, en primer lugar, destacar la lúcida interpretación de lo informal como un territorio poblado por la pugna constante de un equilibrio precario entre los afectos negativos y positivos que lo recorren. En este contexto, y frente a la negatividad que caracteriza a la informalidad, ambos equipos deciden asirse a la afectividad positiva, tratando de develar el potencial que se oculta en las áreas informales.
En este sentido, y siempre a partir de la experiencia de análisis y trabajo compartida con los habitantes de las favelas o los barrios, se inicia la posibilidad de superar el estigma al reclamar la dignidad de cada casa y de cada barrio, dejando de lado la multitud de connotaciones negativas asociadas. Nos encontramos entonces con que el potencial de lo informal desborda a las herramientas tradicionales del urbanismo al apelar a cuestiones profundamente subjetivas y que apelan a la construcción de la identidad, a la identificación con el lugar habitado o a la apropiación de un determinado territorio concreto.

\section{Informalidades afectivas}

La propia focalización de la acción arquitectónica sobre el sustrato que la implicación comunitaria conforma sienta las bases para la posibilidad de una lectura afectiva. Situar el foco de atención sobre los habitantes de un territorio implica empezar a desentrañar la complejidad de las relaciones y de las cualidades sensibles a las que tanto Deleuze como Braidotti se refieren con la idea de territorialidad. Pensar la arquitectura desde el afecto supone, por su parte, reconocer a sus habitantes y ejecutores como piezas fundamentales de la red 
de relaciones que conforman el territorio habitado. En este marco, la ciudad informal es un lugar privilegiado de acontecimientos y de encuentros. Lugar por excelencia de lo imprevisto, las ciudades informales son también lugares de choque, de disputa y de encuentro. Además, las ciudades informales son verdaderos espacios lisos, unos lugares surcados por afectos y abiertos a la transformación constante.

Las políticas públicas de intervención sobre lo informal no pueden -o no deben-ignorar la dimensión subjetiva fundamental sobre la que descansa la vivencia de esta forma urbana no-hegemónica. Los territorios informales componen intrincados tejidos sociales y humanos, en los que el habitar lo urbano se experimenta y se practica de una manera distinta. La incorporación de la subjetividad y de los afectos como materia del proyecto puede ayudar en los necesarios procesos de tránsito derivados de las diferentes actuaciones urbanísticas realizadas sobre estos territorios (Besoain y Cornejo, 2015). Metodológicamente, las entrevistas, los testimonios de vida, las conversaciones, los recuerdos, las fotos y la memoria de los pobladores informales se convierten en una herramienta primordial para comprender estas experiencias intensas del habitar, orientando así la labor de unos arquitectos y técnicos para que no destruyan, con la marca de la normalización, las cualidades de unos territorios de alteridad radical.

Así, preguntarse qué significa habitar una favela o un barrio, preguntarse qué significa habitar una ciudad informal inicia un proceso de reconsideración de la imagen consolidada de unos territorios que han visto su reconocimiento como parte legítima de la ciudad, permanentemente negada. La mirada hacia lo informal desde el afecto permite aferrarnos a unas propiedades poco visibles, una naturaleza oculta que apela a realidades intangibles, como la dignidad, la pertenencia, la apropiación de determinados espacios o la identificación personal con ciertos lugares.

Es cierto que el impacto que la teoría del afecto ha tenido en otras disciplinas parece aún muy lejos de los estudios y la práctica arquitectónica. Sin embargo, también nos parece cierto que, pese a su condición de ciudades de olvido y miseria, las áreas informales parecen estar tendiendo una mano hacia los afectos, para poder desentrañar, mediante su incorporación al ejercicio arquitectónico, unas complejidades asociadas a las experiencias de habitar y producir territorios que nuestra disciplina tantas veces ha olvidado. 


\section{Referencias bibliográficas}

Besoain, C., y Cornejo, M. (2015). Vivienda social y subjetivación urbana en Santiago de Chile: Espacio privado, repliegue presentista y añoranza. Psicoperspectivas. Individuo y Sociedad, 14(2), 16-27.

Braidotti, R. (2009). Transposiciones. Sobre la ética nómada. Barcelona: Gedisa.

Brillembourg, A., Feireiss, K., y Klumpner, H. (Eds.). (2005). Informal city. Caracas case. Múnich: Prestel Verlag.

Davis, M. (2014). Planeta de ciudades miseria. Madrid: Akal.

Deleuze, G. (2015). En medio de Spinoza. Buenos Aires: Cactus.

Deleuze, G., y Guattari, F. (2012). Mil mesetas. Capitalismo y esquizofrenia. Valencia: PRE-Textos.

Anagrama.

Dosse, F. (2009). Gilles Deleuze y Félix Guattari. Biografía cruzada. Buenos Aires: Fondo de Cultura Económica.

Jáuregui, J. M. (2012). Estrategias de articulación urbana. Buenos Aires: Nabuko.

Lefebvre, H. (2013). Il diritto alla città. Verona: Ombre Corte.

McGuirk, J. (2015). Ciudades radicales. Un viaje a la nueva arquitectura latinoamericana. Madrid: Turner Publicaciones.
Parr, A. (Ed.). (2010). The Deleuze dictionary. Edinburgh: Edinburgh University Press.

Spinoza, B. (2015). Ética demostrada según el orden geométrico. Madrid: Alianza.

Turner, J.F.C. (1977). Vivienda: todo el poder para los usuarios. Madrid: H. Blume.

UN-Habitat (2003). The challenge of slums: global report on human settlements. London: Earthscan Publications.

Urban Think-Tank (2013). Torre David: informal vertical communities. London: Springer. 